\title{
Do we Build Similar Molecules for Comorbid Diseases? Tevarud in Drug Design, an Analysis for Depression and Inflammation
}

\author{
F. Esra ÖNEN BAYRAM*[a], Sarah A. A. ALRADHWANI[a] , Gulcin TUGCU[b] and \\ Hande SIPAHI[b] \\ [a] Department of Pharmaceutical Chemistry, Faculty of Pharmacy Yeditepe University, Istanbul 34755, \\ Turkey E-mail: filizesraonen@gmail.com; esra.bayram@yeditepe.edu.tr [b] Department of \\ Pharmaceutical Toxicology, Faculty of Pharmacy Yeditepe University, Istanbul 34755, Turkey
}

\section{Supplementary Information}

Molecule dataset:

The dataset used for the similarity evaluation is provided for both the AD and AI molecules with the corresponding references in annexes.

\section{Similarity calculation:}

The MACCS and Pubchem fingerprints were generated using the PaDEL-Descriptor software (Yap, J Comp Chem, 2011, 32, 1466). Then the cosine coefficient was calculated using the IBM SPSS Statictics V.25 software through the distance calculation. For the determination of the Tanimoto coefficient a Matlab code was used according to the mathematical definition of the coefficient. The lines were adapted from the Mathworks site (https://www.mathworks.com/matlabcentral/fileexchange/13405-tanimoto) and are as follows:

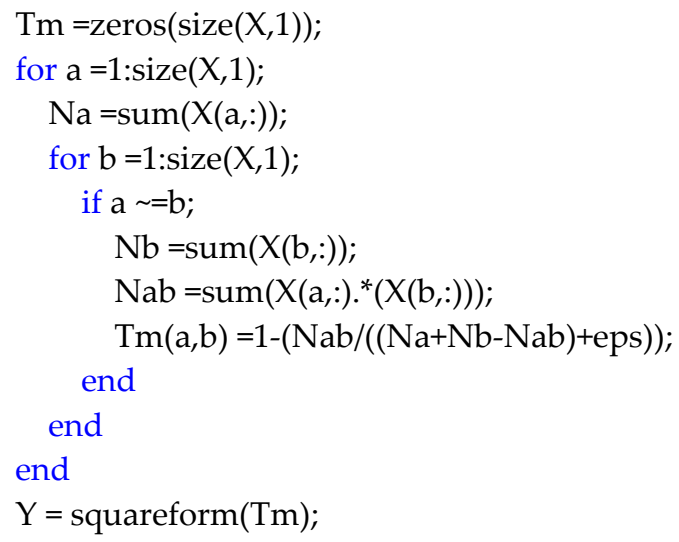

Data analysis:

For the generation of the heatmaps the following procedure was applied to the data obtained for the Tanimoto and cosine coefficients using the MACCS or PubChem fingerprints. Thus 4 different heatmaps were obtained.

For each case, the results were exported to Excel and all the similarity coefficients were gathered in a matrix. Data corresponding to the AD/AI pairs were extracted from the matrix and identified as AIXX AD XX as follows: 


$\begin{array}{lr}\text { AI1 AD1 } & 0.350649 \\ \text { AI2 AD1 } & 0.375 \\ \text { AI3 AD1 } & 0.4375 \\ \text { AI4 AD1 } & 0.263158 \\ \text { AI5 AD1 } & 0.264706\end{array}$

The 19071 values were then sorted from largest to smallest. The pairs that were identified to present more than $90 \%$ of similarity for the Tanimoto coefficients and $80 \%$ similarity for the Cosine were selected (Table 1.). These values were then transfered into a new $\mathrm{AD} / \mathrm{AI}$ matrix and the cells containing values were colored using the "conditional formatting" function of Excel. A 3-color scale was used for generating heatmaps where the red color was chosen for the value of 0 , the green color for the value of 1 and the yellow for the $50 \%$ percentile (Figure 3 ). 